\title{
GENÈSE DES SCIENCES HUMAINES
}

Étienne Pasquier, Les Recherches de la France. Éd. critique avec introd., variantes et notes publ. sous la dir. de Marie-Madeleine Fragonard et François Roudaut, 3 vol. Paris, Honoré Champion, 1996. 14 × 22,5, 2281 p., bibliogr., index (Textes de la Renaissance, $n^{0} 11$ ).

Né en 1529 à Paris, Étienne Pasquier a entrepris des études de droit à partir de 1546. Après avoir suivi l'enseignement de François Hotman dans le quartier latin, de Jean Cujas à Toulouse et de Marianus Socinus à Bologne, il débuta au barreau de la capitale à l'automne 1549. Mais comme il eut peu d'affaires à plaider pendant les six premières années de sa carrière, il passa beaucoup de temps à lire et à écrire au dêbut đes années 1550. C'est ainsi qu'il conçut le projet de ses Recherches de la France, dont il commença la rédaction vers 1555 . Constamment remanié, complété et approfondi jusqu'à sa mort, en 1615, cet ouvrage devint l'un des livres les plus importants de la deuxième moitié du $\mathrm{xv}^{e}$ siècle, tant par son ampleur que par sa notoriété.

Dès l'origine, Pasquier conçut son travail comme une ouvre d'histoire. Dans une lettre à Cujas, il décrivait en effet sa démarche de cette façon : « Je me suis mis à la recherche des anciennetez de la France; et pour cette raison, j'ay appelé mon cuvre Recherches. L'entreprise est de grand labeur, et qui requiert de feuilleter plusieurs livres anciens. " Historien de son temps, soucieux de remonter aux sources, de traquer les origines des institutions, des structures sociales ou du vocabulaire de son époque, Pasquier adopta une méthode profondément originale, à tel point qu'il ne serait pas excessif de voir en lui l'un des pères fondateurs d'une "nouvelle histoire » comprise comme une science humaine devant s'affranchir de tout préjugé.

Il choisit ainsi d'éliminer toutes les informations qui lui apparaissaient comme des légendes dénuées de tout fondement. Très critique envers ses sources, il décida « [...] de ne rien dire qui importe sans en faire preuve, à la charge que, si ceux qui viendront après moy voguent en mesme eau (comme il sera aisé de le faire, la première glace estant rompuë), et me font cet honneur de reconnoistre quelque chose de moy, je la leur donne bien de bon cceur, et veux qu'elle soit estimée leur appartenir, comme si elle estoit de leur tres-fonds ». Conscient que son ouvrage allait devenir une référence pour les recherches futures, Pasquier ne voulut utiliser que des documents qui lui semblaient fiables : non seulement les auteurs antiques, amplement édités par les humanistes depuis plus d'un siècle, mais aussi les grands chroniqueurs 
médiévaux (Grégoire de Tours, Bède le Vénérable, Hincmar, Froissart, Joinville...), le Code justinien, les coutumiers des provinces françaises et les principaux recueils des actes royaux.

De même, il ne cessa de prendre ses distances à l'égard des "opinions communes " de son siècle. Convaincu qu'un lettré devait se méfier a priori des représentations, il voulut combattre les idées reçues, y compris celles qui avaient cours dans ce petit monde de la magistrature qu'il connaissait bien. Il poussa, en effet, le scrupule jusqu'à s'élever contre ses collègues des cours souveraines qui prétendaient jouir d'une noblesse équivalente à celle des gentilshommes ayant suivi la voie des armes. Avec un souci de nuance tout à fait caractéristique de son style, il écrivit ainsi : «Quant à moy, je ne me suis point icy proposé de vilipender les estats de ceux qui suivent la robbe longue, ny generalement de ceux qui sont habituez ès villes clauses : car en ce faisant, serois-je prevaricateur contre moy-mesme. Aussy sçay-je bien que tout homme, en tout estat, qui fait profession de vertu et de vie sans reproche est noble, sans exception. Toutefois, [...] je ne seray jamais jaloux ni marry, qu'à ceux qui exposent leur vie pour le salut de nous tous, soit attribué le tiltre de noble, plustost qu'à ceux qui, dedans leurs palais, à leurs aises, se disent vacquer au bien des affaires d'une justice. "

Pasquier innova, enfin, parce qu'il s'écarta résolument du genre traditionnel que représentait la chronique. Dès la première édition de 1560 , il adopta une structure thématique qu'il ne remit jamais en question dans les développements ultérieurs de son ouvrage. Le premier livre des Recherches fut consacté à la Gaule, à la conquête romaine et aux invasions barbares. Il y enonçait les caractères particuliers à chacun des peuples qui vivaient en France depuis l'Antiquitê, esquissant là une ethnologie rudimentaire qui allait connaître une longue postérité, jusqu'à l'Esprit des lois de Montesquieu. Le deuxième livre, publié cinq ans plus tard, traita de la création des cours souveraines, des grands offices de la couronne et des lois fondamentales du royaume. Pasquier s'attarda tout particulièrement sur le haut Moyen Âge, où il situait l'origine des institutions françaises.

La suite, préparée dans les années 1570-1580, ne parut qu'après les troubles de la Ligue. Dans le livre III, il s'employa à justifier le gallicanisme : sans chercher à y décrire une histoire de l'Église, il voulut démontrer surtout que le pape n'avait aucun droit sur le clergé de France. Le livre IV fut consacré aux fondements de la justice criminelle, et le livre $\mathrm{V}$ aux structures du pouvoir franc. Le livre VI, sans doute le plus hétérogène de tous, exposa les origines de quelques troubles civils, notamment l'affaire du connétable de Bourbon, mais évoqua également quelques personnalités qui intéressaient Pasquier, comme Abélard et Héloïse, Bayard et Charlemagne. Les livres VII et VIII furent plus unifiés. Ils rassemblèrent toutes ses observations sur les origines de la langue française, afin de démontrer qu'elle était tout aussi intéressante et riche que le latin. Dans cette perspective, il ébaucha une première démarche philologique, en cherchant à retrouver la racine de nombreux proverbes et expressions courantes. Enfin, dans le livre IX, il essaya de retracer la naissance de l'Université, afin d'exalter sa vocation éminente, notamment pour l'enseignement juridique.

Cette quête des origines ne fut pas seulement menée comme une vaste entreprise d'érudition coupée de son époque. Bien au contraire, en écrivant ses Recherches, Pasquier entendait justifier les pratiques de son siècle, légitimer certains choix, en 
insistant sur leur ancienneté, garante de leur valeur. Livre d'histoire, son texte n'en était donc pas moins empreint de nombreuses allusions à l'actualité de son temps, qui lui permettaient de faire de fréquents allers-retours entre le passé et le présent. Cette préoccupation constante s'exprima dans les trois principaux centres d'intérêt de son ceuvre : le domaine religieux, le domaine juridique et le domaine politique. Sur le plan religieux, il se fit le farouche défenseur d'un gallicanisme intransigeant. Une telle prise de position n'était pas surprenante, de la part d'un homme de loi du $\mathrm{XvI}^{e}$ siècle. Mais Pasquier voulut s'affranchir des arguments polémiques les moins fondés, en démontrant surtout que l'Église de France avait, depuis toujours, été libre de toute tutelle; les ingérences de la papauté, particulièrement nettes dans les années 1580, étaient donc pour lui des nouveautés insupportables. Dès 1564, comme bon nombre de magistrats du Parlement, il avait ainsi lutté contre l'introduction des Jésuites dans le royaume, car il y voyait un danger pour l'indépendance du clergé et la prérogative royale.

Héritier des légistes du $x_{1} v^{e}$ siècle qui avaient énoncé la règle selon laquelle le souverain était empereur en son royaume, Pasquier tenta pourtant de promouvoir, dans le champ juridique, l'idée d'un nécessaire équilibre entre la couronne et les grands corps de l'État. À la suite de Claude de Seyssel, il décrivit les cours souveraines comme les gardiennes des lois fondamentales qui empêchaient le roi de se muer en tyran. Dans cette perspective, l'enregistrement des édits et des ordonnances par le Parlement n'était pas seulement l'expression d'un simple contre-pouvoir, car dans son esprit, cette pratique participait aussi à la grandeur du souverain et conférait au gouvernement davantage d'efficacité : selon lui, il était « digne de la Majesté d'un Prince que nos Roys (ausquels Dieu a donné toute puissance absolue) ayent d'ancienne institution voulu réduire leurs volontez sous la civilité de la loy [...] Dès lors que quelque ordonnance a esté publiée et vérifiée au Parlement, soudain le peuple françois y adhère sans murmure, comme si telle compagnie fust le lien qui noüast l'obeyssance des sujets avec les commandemens de leur Prince ".

Après avoir été nommé avocat général à la Chambre des comptes, en 1585, Pasquier resta fidèle à la couronne. Il aurait pourtant pu prendre parti pour la Ligue, comme beaucoup de ses collègues, d'autant qu'il était lié à la clientèle des Guises. Il préféra s'abstenir, car si les Recherches prônaient l'idée d'un pouvoir central tempéré par la loi, elles traduisaient également une inébranlable confiance dans la souveraineté royale. Du point de vue politique, il se situait en effet dans la vieille tradition des Miroirs des princes, où la plupart des auteurs qui l'avaient précédé dans cet exercice ne cherchaient que très rarement à remettre en cause l'étendue de la puissance du monarque. Il est vrai que Guillaume Budé, dont L'Institution du prince était parue en 1547, avait été plus radical que lui, puisqu'il estimait que le roi, seul dépositaire de la loi divine, pouvait gouvemer selon son bon plaisir. Mais Pasquier consacra de longs développements à décrire les désordres causés dans le royaume par la folie de Charles VI, en insistant aussi sur les problèmes qui étaient posés par les minorités et les périodes de régence. Éclairé par Dieu, le roi devait faire un usage mesuré de son pouvoir afin de contraindre les aristocrates à lui obéir. Concluant à propos des démêlés qui opposèrent le connétable de Bourbon à François $\mathrm{I}^{\mathrm{er}}$, il estima ainsi que cette affaire devait « servir de leçon, et au sujet, et au Prince souverain. À cestuy-là pour luy enseigner que, quelque grandeur qui soit en luy, il n'y a rien qu'il doive tant craindre que de se faire redouter et craindre par son Roy; et au Prince 
souverain, que sur tout, ores qu'il le puisse d'une authorité absoluë, toutesfois il se doit bien donner garde de faire mourir un sien subject sans connoissance de cause, et comme l'on dit, d'une mort d'Estat ».

Les Recherches de la France n'avaient jamais fait l'objet, jusqu'à présent, d'une publication critique. L'édition de 1665 , utilisée ici, a été choisie parce qu'elle représentait l'état le plus achevé d'une œuvre qui ne cessa d'être remaniée par Pasquier, avant d'être complétée par ses héritiers à l'aide de ses manuscrits personnels. L'équipe animée par Marie-Madeleine Fragonard et François Roudaut a accompli un travail considérable, en écrivant des notes permettant d'expliquer certains passages, de corriger d'éventuelles erreurs, de restituer des variantes. Un index très précis permet de se repérer facilement dans ces trois gros volumes, qui ont été enrichis par une liste des lectures de Pasquier, du plus haut intérêt pour faire l'inventaire de ses sources et de ses influences. Il s'agit donc d'une publication fondamentale, qui fera date dans l'historiographie du $x^{e}{ }^{e}$ siècle.

Laurent BouRQuIN

Colas Duflo, Le Jeu. De Pascal à Schiller. Paris, Presses universitaires de France, 1997. 11,5 × 17,5, 128 p. (Philosophies, 94).

Dès lors que la philosophie nous aide à nous comprendre et à comprendre le monde, le jeu, présent dans notre vie comme sollicitation et comme pratique, peut être un objet de méditation. Colas Duflo montre pourquoi et en quoi le jeu peut intéresser la réflexion philosophique.

Cet intérêt ne s'est pas toujours imposé. Le jeu a longtemps été considéré comme une activité réservée aux enfants ou comme un pur divertissement. Aristote, par exemple, le disqualifie, lui dénie toute possibilité d'être une fin suprême car le bonheur ne peut être que le résultat d'une activité conforme à la vertu, et non d'un divertissement. Or le jeu n'est ni une fin, ni une activité au plein sens du terme. Thomas d'Aquin, quant à lui, attribue certes deux vertus au jeu, le repos et l'urbanité, mais elles sont assujetties à une pratique mesurée du jeu qui doit rester un délassement contrôlé par la raison.

Duflo montre ainsi que le jeu ne commence à être véritablement pris au sérieux qu'à dater des XvII et ${ }^{e}$ XvII $^{e}$ siècles et que cette mutation peut être considérée comme achevée avec Friedrich Schiller.

Une première partie met en évidence le thème du jeu dans les textes théoriques et retrace le parcours philosophique qui conduit les auteurs du xvir' siècle à fonder une anthropologie qui rend nécessaire l'intervention du thème du jeu. La réflexion morale est naturellement amenée à se préoccuper de la valeur du jeu. Les pouvoirs temporels, toujours encouragés par l'Église chrétienne, tentent de l'interdire ou de le réglementer, lorsqu'il s'agit de jeux de hasard et d'argent. En effet, le jeu est socialement répréhensible et nuisible à la morale publique puisque l'argent n'y a plus de sens que comme enjeu.

Cependant, au $\mathrm{XvI}^{\mathrm{e}}$, et surtout au $\mathrm{XvII}$ siècle, le mathématicien découvre dans l'activité ludique un terrain d'analyses nouvelles : les jeux de hasard et le «pro- 
blème des partis ». Le jeu conquiert alors la dignité d'un phénomène né de l'ingéniosité humaine, méritant par là l'attention du savant. Le philosophe Leibniz envisage un traité général des jeux qui nous donnerait de précieux renseignements sur l'art d'inventer et le fonctionnement de l'esprit humain libre de contraintes. Les calculs, les stratégies, le fait d'essayer de deviner l'adversaire, tout cela se rapproche de l'activité du savant tentant de percer le mystère de la nature. Le jeu est fondamentalement lié à la probabilité et à l'activité de prévision.

Avec la " géométrie du hasard ", Pascal, en moraliste et en savant, fait apparaître l'idée d'une justice du jeu. Le hasard, échappant à son inationalité suspecte, n'est plus simplement lié à un arbitraire sans règle comme il pouvait l'être encore dans les textes des moralistes chrétiens ou les discours des directeurs de conscience. C'est également en observateur de la condition humaine que Pascal s'intéresse au jeu, avec sa célèbre analyse du divertissement. Le joueur trouve son plaisir dans l'étourdissement de ne pas penser. Si le divertissement est condamnable, c'est qu'il parvient à ses fins. Enfin, Pascal propose son fameux pari, jeu suprême qui reconduira le joueur à Dieu. Il s'agit d'utiliser les mécanismes du jeu pour nous ramener aux considérations dont le jeu nous éloignait.

Dans une deuxième partie, Duflo dresse un « autoportrait du joueur au xvII" siècle " et soutient que le Siècle des lumières mériterait d'être nommé le « siècle du jeu », ce dont Casanova offre un excellent témoignage. La préférence du Chevalier va aux jeux de hasard et d'argent dans lesquels la seule alternative est de gagner ou de perdre. Le jeu décide, selon la bonne ou la mauvaise fortune, d'une destinée, d'un voyage, d'une aventure.

Le témoignage de l'aventurier montre que le jeu se généralise dans tous les pays et dans toutes les classes sociales de l'Europe, souvent d'ailleurs ent dépit des interdictions qui ajoutent au plaisir du joueur, nécessairement tricheur et conduit à « corriger la fortune ". Casanova fait explicitement le lien entre séduction et jeu. Il ne s'agit toujours que de fuir à tout prix l'ennui.

Les articles de l'Encyclopédie où se rencontre le jeu peuvęnt alors dresser un état des lieux. Le critère du hasard permet une classification. La condamnation des jeux ne vient plus de l'injustice de ce même hasard. Du point de vue du contrat, le jeu est parfaitement légitime. C'est l'excès auquel il entraîne qui est condamné.

Ce point fait, Duflo, dans la troisième partie de l'ouvrage, entreprend de montrer qu'avec la naissance progressive de l'intérêt poar l'enfant se précise l'idée que l'étude puisse prendre la forme du jeu. Dans l'éducation telle que la prône Rousseau, le jeu, bien compris comme une activité, n'est toléré qu'à condition que le plaisant soit aussi utile. S'opposant souvent à Rousseau, mais tenant grand compte des thèses du philosophe genevois, Kant soutient qu'il est impossible de fonder une pédagogie sur l'apprentissage par le jeu. En effet, le jeu est agréable alors que le travail est désagréable et contraignant. Il n'en faut pas moins apprendre à travailler pour être libre. Cependant, dans le jeu, les enfants se soumettent à des règles qu'ils ont libtement choisies et font ainsi l'apprentissage de l'autonomie de la volonté, principe suprême de la moralité. Le jeu trouve alors sa place dans une anthropologie qui associe la liberté, la légalité et la possibilité de comprendre activement la nature, et non de connaître passivement une harmonie prétablie. De plus, cet accord dans l'homme est lié à un accord entre les hommes. Le jeu est un facteur d'universalité. 
Ia quatrième partie de l'ouvrage expose en quoi Schiller téalise la synthèse de ce mouvement de réhabilitation du jeu qui s'étend sur deux siècles. Dans les Lettres sur l'éducation esthétique de l'homme, publiées en 1795 , le thème du jeu contraint la philosophie à dévoiler sa conception de l'homme, fondée sur la double nature sensible et raisonnable de celui-ci. L'homme est d'abord un être de nature, mais pas seulement. Il convient de distinguer l'homme physique de l'homme moral, Le problème que pose l'anthropologie consiste alors à trouver un troisième caractère qui concilie ces deux natures de l'homme. Les Grecs avaient montré que cet homme « réconcilié " était possible, mais avaient également souligné le déchirement entre tendance sensible et tendance formelle, véritable noud de forces contradictoires. Pour Schiller, c'est la tendance au jeu qui va permettre de constituer l'homme total. Cette tendance n'est pas un instinct, mais le résultat de la coopération harmonieuse du sensible et du formel, une idée régulatrice, un but toujours à atteindre, la réconciliation des opposés. Son objet, soutient Schiller, n'est autre que la beauté.

Pourquoi nommer a tendance au jeu * ce qui désigne une * tendance au beau *? Le jeu intervient parce qu'il est considéré comme un vecteur d'barmonie, donc de beauté et d'équilibre. Il permet de faire passer la sécheresse des principes dans la sensibilité et témoigne empiriquement de capacités propres à instaurer une légalité qui ne soit pas ressentie comme une brimade. Le jeu, que Schiller admet également chez les animaux, est cependant la marque spécifique de la liberté humajne dégagée de l'instinct. C'est le lieu de la complétude de l'homme. L'un de ses synonymes est, par exemple, l'amour : l'homme y est total, entier, c'est-à-dire humain.

L'ouvrage de Duflo, petit par le volume, est d'une grande densité. Il contient en appendice trois textes, l'un de Leibniz, les deux autres de D'Alembert et de Kant, qui marquent, chacun à sa manière, des étapes de cette histoire convaincante de la prise en compte du jeu dans la philosophie, histoire qui conduit une pratique reléguée comme mineure à la dignité d'un concept fondateur de l'anthropologie philosophique. Il était important de nous éclairer sur les raisons et le déroulement de cette étonnante évolution.

Jean-Marc RoHRBASSER

Philippe Steiner, La * Science nouvelle * de l'économie politique. Paris, Presses universitaires de France, 1998 . 11,5 × 17,5, 128 p., glossaire, bibliogr.

Le plus jeune disciple de François Quesnay (1694-1774) et son premier éditeur, Pierte-Samuel Dupont de Nemours, est également le premier historien de la physiocratie, et la terminologie et la démarche qu'il adopte pour en retracer la naissance et le développement, va durablement marquer ses successeurs. $\dot{A}$ tort et à raison, selon Philippe Steiner : à taison, lorsqu'il qualifie le discours physiocratique de * Science nouvelle " de l'économie politique, à tort, lorsqu'il en lie l'origine par trop étroitement au concept du droit naturel, notamment en plaçant en tête du recueil Physiocratie (1767), le texte, tardif, de Quesnay intitule justement « Droit naturel » (1765). Ce demier point annonce que l'auteur se démarque ici de travaux récents sur ce 
sujet, en particulier l'ouvrage de Catherine Larrère, L'Invention de l'économie au xvII siècle (Paris, Presses universitaires de France, 1992).

Pour caractériser - et comprendre - le nouveau discours sur le social que constitue la physiocratie, l'auteur propose de s'attacher aux éléments suivants : 1) qu'est-ce que la science pour Quesnay ? 2) comment cette conception se traduit-elle lorsqu'il se saisit de la question de la liberté du commerce des grains, puis lorsqu'il conceptualise l'ensemble de l'activité économique? 3) la place que Quesnay accorde dans la sphère politique à l'espace public, ou «sphère publique bourgeoise »; 4) sa conception large de l'économie politique faisant de celle-ci une partie des sciences morales et politiques. Cependant, ces éléments sont inégalement traités : ce sont surtout les deux premiers qui forment le coeur de l'ouvrage et soustendent ses trois premières parties ( (Economie, science du commerce et économie politique ", « Sensualisme et ordre naturel », « Analyse économique de l'ordre naturel »), tandis que les derniers sont abordés dans une courte quatrième partie et en conclusion.

L'apparition de la " Science nouvelle » date du milieu du Xviı" siècle. Qu'entendt-on par «science » à l'époque? Steiner souligne que, jusqu'en 1750 environ, la définition de la science - et des sciences - reste vague : elle se précise avec D'Alembert, et surtout Condillac (que Quesnay connaît dès 1749), qui distinguent alors clairement la science de la religion et de la métaphysique. Plus intéressant, ce statut de science a-t-il été revendiqué dans le domaine économique avant Quesnay? Deux projets antérieurs à la physiocratie ont une telle ambition : la « science du commerce », dont François Véron de Forbonnais développe les principes, et la "science ceconomique » proposé par Carl von Linné dans le Journal aconomique dès 1752. Le premier est désormais bien connu (mais il n'aurait pas été inutile d'y. attacher plus d'importance), ce qui est loin d'être le cas du second, et il faut regretter que Steiner n'ait pas le loisir ici de le présenter plus en détail. Ainsi la « Science nouvelle " n'est-elle pas sans concurrente, ce qui ne doit pas être négligé pour comprendre les voies qu'elle choisit pour se différencier et s'affirmer. Dans les premiers textes économiques de Quesnay, les articles « Fermiers » (1756) et « Grains » (1757) parus dans l'Encyclopédie, nulle allusion n'est faite à une nouvelle science. Il faut attendre la Philosophie rurale (1759), et donc la collaboration avec Mirabeau, pour que le Tableau économique du maître soit présenté comme «le précis et la base de la science économique ". Cette dernière se distingue par un aspect remarquable, mis en exergue : l'importance accordée aux «calculs", qui depuis a été abondamment relevée. L'auteur note, à ce sujet, qu'à la différence de Malebranche - "son supposé mentor en philosophie " - Quesnay, qui n'attribue pas. aux calculs une validité illimitée, récuse toute "mathématique des imperceptibles ", entre autres, le calcul différentiel. Autre trait marquant, l'attention portée par Quesnay au langage et à la clarification conceptuelle qui rappelle celle de Condillac (et là encore le démarque de Malebranche).

La deuxième partie de l'ouvrage est sans doute la plus novatrice. Steiner y insiste sur la nécessité de mettre au jour la nature des liens entre la « science nouvelle » et la théorie de la connaissance de Quesnay, et suggère deux pistes pour y parvenir : d'une part, Quesnay, lecteur de Locke, est marqué par la philosophie sensualiste, renouvelée au même moment par Condillac; de l'autre, Quesnay étant également influencé par la philosophie cartésienne, il convient de reconsidérer ce qu'il a 
emprunté à Malebranche. Ainsi Steiner rejette au second plan les concepts de droit naturel et d'ordre naturel : chez Quesnay, la réflexion sur la connaissance serait première dans l'ordre de la science. La démonstration s'appuie principalement sur deux textes du physiocrate : le « Discours prêliminaire » de l'Essai physique sur l'aconomie animale ( ${ }^{2}$ éd., 1747) et l'article * Évidence » de l'Encyclopédie (1756), tous deux faisant transition entre son ouvre médicale et son cuvre économique. Dès 1747, Quesnay opte pour une conception sensualiste de la connaissance, qu'il développe dans «Évidence » et réaffirme à la fin de sa vie dans les Recherches philosophiques sur l'évidence des vérités géométriques (1773) : il s'y oppose ainsi directement à Descartes et à Malebranche, en considérant que non seulement les sens sont la source de nos connaissances, mais encore le seul moyen de corriger nos eneurs. Par ailleurs, le fait que la conception de l'ordre naturel économique chez Quesnay n'ait pas de racines théologiques relativise encore, aux yeux de l'auteur, l'importance habituellement accordée à Malebranche pour comprendre l'œuvre du physiocrate. Et pour étayer cette hypothèse, sont invoquées toutes les « occasions manquées " où Quesnay aurait pu avoir recours au philosophe; mais le fruit d'une telle démarche, pour récuser une filiation intellectuelle, pourra être constesté. Quesnay aurait surtout emprunté à Malebranche une conception cartésienne de la science et de la méthode. Puis il est examiné comment se concilient cartésianisme et sensualisme, autrement dit ordre naturel et sensualisme qui constituent ce que l'auteur dénomme le "sensualisme normatif » de Quesnay. Il en ressort que ce dernier ne peut être assimilé aux concepteurs de systèmes fondés sur des hypothèses, du moins dans ses premiers écrits : la base factuelle, voire expérimentale, de ses travaux doit être reconnue comme telle. Cependant, dans les remarques qui accompagnent le Tableau économique, Quesnay affirme aussi que ses principes théoriques sont vrais indépendamment de la base factuelle retenue. Steiner relève que cette seconde orientation se renforce dans les textes ultérieurs du physiocrate, surtout après 1765 : malheureusement, il note ici cette évolution sans en retracer les étapes et en analyser plus avant les fondements.

L'analyse économique de l'ordre naturel est ensuite l'objet d'une approche originale et d'importants développements. 'Ainsi, pour n'en évoquer que quelques-uns, Steiner montre que la place accordée par Quesnay au comportement des agents économiques est bien plus capitale qu'on ne le reconnaît habituellement. Or, le comportement des agents est justement ce qui permet de faire le lien entre sa théoric de la connaissance et un élément décisif de sa théorie économique. La plupart des agents agissent selon leur intérêt immédiat et leur liberté animale, et ont ainsi des actions régulières dont on peut faire la théorie. S'il y a opposition d'intérêt, la concurrence est alors essentielle pour la régler : le débat contradictoire qu'elle instaure entre les agents représente l'essence de l'ordre naturel économique tel que le conçoit Quesnay. Pour que le bon prix, notamment celui des grains, la rente du propriétaire, le salaire s'établissent à des montants satisfaisants pour l'ensemble du corps social, il faut s'en remettre au comportement intéressé d'individus qui débattent contradictoirement de leurs intérêts sur un marché concurrentiel. Cependant, dans certains cas, la confrontation d'intérêts opposés entre deux groupes, le conflit entre les libertés animales, ne peuvent être résolus sans le recours à la liberté d'intelligence, qui n'est pas à la portée de tous les agents : seul le philosophe, dans un premier temps, la possède et peut fournir la solution. La répartition du produit 
net, attribué finalement en totalité aux propriétaires, chargés en retour de payer l'impôt unique, a pour conséquence que ceux-ci doivent également, comme le philosophe, maîtriser la a Science nouvelle * et user de leur liberté d'intelligence.

La notion de gouvernement économique est un fil conducteur dans l'auvre de Quesnay. En cela, ne s'inscrit-il pas dans une longue tradition de «conseillers du prince $n$, ce dont il aurait peut-être fallu mieux rendre compte ? L'objectif physiocratique est de restaurer la bonne marche du royaume agricole, dont la situation s'est dégradée, d'une part, en raison de la méconnaissance de l'ordre naturel économique, de l'autre, du fait des pressions néfastes qu'exerce le "système des commerçants ". Quesnay construit donc une opposition entre deux formes d'organisation économique, et ainsi deux gouvernements économiques, la république commerçante et le royaume agricole, opposition qui tient d'abord à des critères géo-économiques (exemple de la Hollande et de la France). Il en conclut d'abord qu'il existe une complémentarité et une coopération possible entre ces deux types de pays (et rejette ainsi toute vision belliqueuse des échanges internationaux si commune au siècle précédent) et ce faisant, comme le remarque Steiner, ne jette nullement la pierre à ceux voués au négoce où le commerce dégage un produit net. La principale erreur de Colbert et de ses successeurs protecteurs des marchands fut d'appliquer au royaume agricole des règles propres à la république commerçante. Finalement, pour rếtablir le royaume agricole, il faut connaître les lois de l'ordire naturel économique - le Tableau économique - et le gouvernement économique doit se réduire à peu de choses, à laisser faire. L'auteur souligne que, sous cet anglc, il y a clairement chez Quesnay autonomie de la sphère économique vis-à-vis de la sphère de décision politique. Est-ce conciliable avec des interprétations récentes de la physiocratie, appréhendée comme un projet visant à a convertir le politique en économié "?

Dans une dernière partie, il est question des notions de despotisme légal et d'espace public. Elles découlent de la nécessité pour Quesnay, une fois définis l'ordre naturel économique et le gouvernement économique le plus en adéquation avec celui-ci, d'envisager comment ce gouvernement économique peut être instaurer et préserver. Le lien entre le politique et l'économique s'établit autout des rapports entre la Science nouvelle, le souverain et l'opinion éclairée. Deux conclusions de l'auteur retiennent ici l'attention. Dans le seul texte qu'il consacre spécifiquement au \& Droit naturel *, Quesnay se révèle hésitant, voire imprécis, lorsqu'il s'agit de le définir, en particulier dans la terminologie qu'il adopte, lui si soucieux d'ordinaire du langage. Quelle portée faut-il dès lors accorder à ce texte tardif? En second lieu, le problème de la connaissance est présenté comme un aspect essentiel pour pénétrer la réflexion politique de Quesnay. Ainsi, le lien politique peut se définir par l'intermédiaire de la diffusion et de la circulation de la connaissance des lois de l'ordte naturel, et si on associe cette thèse à celle mettant l'accent sur la propriété, selon Steiner, la Science nouvelle « prend place dans ce que J. Habermas a qualifié de sphère publique bourgeoise où se rassemblent les individus susceptibles de faire un usage public de leur raison, en matière politique notamment, mais pas seulement; individus désignés par leur qualité de propriêtaire d'une part, par leur raison, d'autre part $»$ (p. 112). D'où, s'il y a bien chez Quesnay une réduction économiciste du politique, il ne faut pas négliger qu'il fait de l'opinion publique une donnée politique fondamentale, et qu'il conserve au politique une place certes limitée mais 
réelle, puisqu'elle est à la fois pédagogie et " contrôle exercé par ceux qui savent sur ceux qui traduisent le savoir en décision $»$ (p. 116).

L'ouvrage livre donc de nouvelles clefs pour comprendre les fondements de la physiocratie. La référence nécessaire au sensualisme est très convaincante. L'appréciation des rapports à établir avec la philosophie de Malebranche présente plus de difficultés, toutes ne sont pas résolues. Quant aux évolutions, voire aux contradictions, relevées dans l'œuvre de Quesnay, elles incitent à revenir sur la chronologie des textes et leurs conditions d'élaboration, à ne pas négliger une perspective plus historique, à étudier enfin les relations que Quesnay a entretenues avec ses « disciples ", et entre autres, à évaluer dans quelle mesure il a pu s'opérer une influence réciproque - et non unilatérale - entre le maître et ses élèves. Autrement dit, $L a$ * Science nouvelle " de l'économie politique, entre autres mérites, devrait stimuler les recherches sur l'histoire complexe de la physiocratie et du discours quesnaysien.

Christine ThÉRÉ

Nature, histoire, société. Essais en hommage à Jacques Roger. Rassemblés et présentés par Claude Blanckaert, Jean-Louis Fischer et Roselyne Rey. Paris, Klincksieck, 1995. 15, $5 \times 24,454$ p., bibliogr., index.

Publié avec le concours du Centre national du livre et de la fondation SingerPolignac, cet ouvrage collectif réunit vingt-sept contributions - dont plusieurs traduites - de collègues et d'anciens élèves de Jacques Roger, qui se veulent l'expression de l'étendue des questions que celui-ci aborde dans ses publications.

Un premier groupe de textes rassemble les études portant sur les concepts, les mots ou les attendus philosophiques qui sous-tendent la signification des grandes notions organisatrices de la pensée scientifique, ou servent à en imaginer la restructuration en vue d'élargir sa communication à tous les hommes, comme Francis Bacon (Advancement of learning, 1605) et Marin Mersenne (l'Harmonie universelle, 1636-1637) en conçurent le projet. Cette première série d'analyses est introduite par une tentative de déconstruction critique de quelques « mythes méthodologiques * (Mirko D. Grmek) obscurcissant notre compréhension de la double composante évolutionniste (l'affinement croissant des données empiriques considérées) et révolutionnaire, par renversement de paradigme, indissociablement à l'auvre dans les processus de la découverte scientifique. Mais, non moins cruciale que la détermination de la dynamique réelle du savoir, est pour l'épistémologue la clarification des présupposés de la sémantique de la science. Ainsi, Vincent Cappelletti relève-t-il l'incapacité de la raison scientifique contemporaine à opérer la synthèse des concepts de mouvement - revêtant pour nous une acception strictement mécanique -, de transformation (metabole), d'évolution et d'information (Einbildung), dans un contexte où la nature a cessé d'être conçue comme «l'acte de la mobilité en devenir [...] vers l'acte de l'acte : la Pensée, dotée de vie et désirée par le monde $\gg$ (Aristote, Métaphysique).

Lorsque l'acte de synthèse conceptuelle relève non plus seulement d'une nécessité d'ordre heuristique, mais constitue le fondement du discours métaphysique, son 
produit s'affirme comme théorie explicative dont la plausibilité reste à démontrer. Ainsi, François Duchesneau se pose-t-il la question de savoir comment la « chaîne des êtres » leibnizienne, dont la structure est de type mathématique, peut faire droit à la versatilité d'une nature contingente. La réponse semble tenir en l'affirmation d'une «conception dynamique de la nature » (Jacques Roger) présupposant l'absence de toute causalité évolutive initiatrice de l'apparition de formes nouvelles par « dérivation » de formes plus anciennes.

L'analyse de l'écho rencontré par l'œuvre de Newton dans la pensée philosophique du XviI ${ }^{e}$ siècle (voir la Lettre sur les aveugles de Diderot), puis de Darwin dans la pensée scientifique moderne, fait l'objet des interventions sur lesquelles se clôture la première partie de l'ouvrage. John Greene tente ainsi d'évaluer l'impact de la « révolution darwinienne » dans l'ensemble des sciences (physique, biologie du comportement, anthropologie, psychologie, sociologie, etc.), sur la philosophie et notre " vision " du monde. Constatant l'importance du nombre de termes employés métaphoriquement par la biologie évolutionniste moderne (l'« effort $»$, le « but $»$, la "valeur »), il soutient que la question fondamentale que pose aujourd'hui encore l'œuvre de Darwin est de savoir si l'idée de nature peut faire sens indépendamment de celle d'un «bien " inhérent au processus de la sélection naturelle.

La deuxième partie de l'ouvrage, qui analyse la constitution et le développement des sciences de la vie, se subdivise en deux sections. La première fait place à des considérations d'ordre philologique (autour de la notion de contagion) ou portant sur l'écart existant entre l'expérience subjective du malade (Maine de Biran) et le témoignage «objectif » des médecins (Moreau de La Sarthe et Laennec) subtilement analysé par Jean Starobinski. Elle se conclut sur l'histoire de l'introduction en France des théories darwiniennes et de la génétique des populations dans les années 1930, puis, de la génétique, et avec la discussion des preuves épidémiologiques aujourd'hui disponibles en faveur de la sélection naturelle (Bertardino Fantini). Le cơur des développements contenus dans cette section se rapporte à la mise en lumière du rôle central joué par la théorie de la génération dans l'ceuvre d'Érasme Darwin, et des limitations imposées par le paradigme mécaniciste et matérialiste de la biologie postdarwinienne aux tentatives faites pour rendre théoriquement compte de la possibilité de rencontres aléatoires entre caractères indépendants, au fondement de la connaissance des lois de l'hérédité. Roselyne Rey montre en quoi la théorie transformiste énoncée dans l'ceuvre d'Érasme Darwin cherche à résoudre de façon unitaire, pour les mondes végétal et animal, le liẹn entre le développement individuel et la transformation des espèces, et ne peut se comprendre en dehors d'une réflexion sur le problème de l'origine de la forme, «traduction et résultante d'un ensemble de mouvements vitaux ou intellectuels $*$. Charles Lenay décrit, enfin, la manière dont Hugo de Vries, s'appuyant sur la double hypothèse de la pangenèse de Darwin et de la continuité du plasma germinatif d'August Weismann, tente de forger une théorie de l'organisation des êtres vivants qui diffère à la fois du préformationnisme classique et de l'épigénétisme causal (mécaniste) présupposant la liaison des caractères entre eux.

Les contributions contenues dans la seconde section abordent un ensemble de questions aussi variées que celles du rapport entre science et idéologie, de la définition des étapes et des modalités de l'identification, de l'unification et de la délimita- 
tion du champ de la biologie, de la constitution d'une approche alternative de la pensée de Lamarck faisant l'économie des présupposés d'une historiographie évolutionniste ou transformiste (Michael Jonathan Sessions Hodge), enfin, de la redéfinition de la nature du processus de l'évolution au $x^{e}$ siècle, dans l'œuvre du néolamarckien René Jeannel (1879-1965). La premiẻre question fait l'objet d'instructives considérations sur l'enchevêtrement des motifs idéologiques (le conservatisme politique) et des raisons théoriques (la lutte contre le matérialisme et le transformisme) expliquant le réinvestissement du cadre conceptuel foumi par l'anatomie philosophique allemande - supposée faire appel à des notions de hiérarchie, de transcendance et d'antimatérialisme et permettant de rapprocher des points de vue apparemment inconciliables de la téléologie et du mécanisme - par les enseignants du Collège royal de chirurgic de Londres, dans les années 1820 , sous l'impulsion de Joseph Henry Green (1791-1863), disciple du philosophe Samuel Taylor Coleridge. L'intérêt du lecteur ne pourra également manquer de se porter sur l'excellente contribution de Giulio Barsanti décrivant le rôle décisif joué par la conception lamarckienne de la vie dans l'autonomisation de la biologie par rapport à la physique et à l'histoire naturelle. La recherche d'une loi générale valant comme clé de la compréhension de la nature vivante conduisit Lamarck à doter toutes les disciplines d'étude de celle-ci d'un cadre unique de référence. La définition des vivants par opposition aux « corps bruts » devait, enfin, conduire la biologie nouveau-née à la certitude que ses objets différaient de tous les autres, qu'il existait un niveau de la réalité irréductiblement biologique.

C'est avec l'appréciation du conditionnement exercé sur la réception des idées de Darwin par l'idéologic multiséculaire de la vie comme effort impliquant douleur et sacrifice, et le regard jeté sur la postérité du concept spencerien de " survie des plus aptes $»$, que se conclut cette deuxième partie de l'ouvrage. Antonello La Vergata tente ainsi d'expliciter les présupposés idéologiques de la conception de l'effort par les thériciens de la biologie de la seconde moitié du $\mathrm{XIX}^{\mathrm{c}}$ siècle, non seulement comme facteur "nécessaire" et "supérieur " de toute transformation (Armand Sabatier), mais encore comme composante indissociable de toute forme de «lutte pour l'existence " (Alfred Russel Wallace, John Arthur Thomson).

Le concept spencerien de survival of the fittest (« survie des plus aptes ») - que Darwin accepta d'employer comme synonyme de la notion de " sélection naturelle * dans plusieurs passages des dernières éditions de L'Origine des espèces -, dont provient l'expression fitness to survive ( "aptitude à survivre ") ou fitness, devint, au début du $x x^{e}$ siècle, le nom d'une grandeur mesurable, le concept d'une « valeur sélective * mathématiquement exprimable. Cette "métamorphose quantitative » sera elle-même à l'origine de l'émergence du concept modeme de fitness, définie comme succès reproductif moyen d'un individu dans une population, dans l'ceuvre d'Alfred James Lotka (1880-1949), et comme produit de la viabilité et de la fécondité, composantes de l'« aptitude » générale d'un organisme chez Ronald Aylmer Fisher (1890-1962). La fitness, ainsi entendue, offre une compréhension des implications démographiques du principe darwinien de la sélection naturelle, que pouvaient difficilement accepter les doctrines eugénistes de Francis Galton et Karl Pearson, et sert de support à une vision philosophique foncièrement optimiste de I'évolution chez les darwiniens du $x x^{e}$ siècle. 
Les textes de la troisième partie de l'ouvrage offrent divers aperçus sur les modes de relation de la science avec son contexte social, philosophique et culturel, entre la seconde moitié du xviı ${ }^{e}$ siècle et la première partie du siècle suivant. Dans son atalyse du contexte culturel de réception des acquis du progrès de la connaissance scientifique au xvm" siècle, Andreas Kleinert démontre l'existence d'une importante dénivellation conceptuelle entre science en cours d'élaboration et science vulgarisée, contredisant l'intention des vulgarisateurs d'alors et l'appréciation optimiste de certains historiens des sciences. De même, comme le rappelle Laurent Loty, repositionner l'histoire de la connaissance dans une histoire culturelle, engage une évaluation du degré d'autonomie des sciences relativement à la métaphysique. Ce que vient à propos confirmer l'exemple de l'ouvre de Dupont de Nemours (1739-1817) s'opposant à la représentation classique d'une coupure entre l'homme et l'animal, réfutant l'innéisme (mémoire Sur l'instinct) et soutenant, à la différence des idéologues, que la psychologie découle des comportements sociaux.

Le mode de relation particulier de la culture avec la science, que les poètes (Louis de Fontanes, Charles Chênedollé, André Chénier) surent instaurer autour de la Révolution française et au début du $\mathrm{XIx}^{\mathrm{c}}$ siècle (Jacques Delille, Népomucène Lemercier), se trouve en dernier lieu finement analysé par Jean Dhombres.

Les articles contenus dans la partie finale du livre abordent en premier lieu la question du rapport des sciences française et américaine dans deux auvres aussi diverses par leur inspiration et éloignées dans le temps que celles du naturaliste français Ambroise M. F. J. Palisot de Beauvois (1752-1820) et de Paul Broca, fondateur de la Société d'anthropologie de Paris (1859), qui défendit contre ses pairs les thèses polygénistes (affirmant l'existence d'une hiérarchie des « types de l'humanité ») de Samuel George Morton, Josiah Clark Nott et George Robins Gliddon, cautionnant l'esclavage des Noirs, au mépris des lois du contrôle savant. L'un des aspects de l'imposture idéologique découlant de ce parti pris consiste en l'affirmation du caractère moralement préférable du concept d'inégalité des races à celuj de leur dégénérescence, induisant malédiction et démérite (Broca, « Recherchess sur l'hybridité $m, 1877)$ !

Deux ultimes interventions s'attachent à mettre en lumière les enjeux d'ordre à la fois culturels, philosophiques et sociopolitiques de. la constitution de la préhistoire et de la paléontologie humaine. Nathalie Richard attire ainsi notre attention sur le fait que l'épistémologie de la préhistoire naissante, au xIx siècle, « intègre [...] un discours matérialiste et transformiste et certains mots d'ordre nationalistes ». Goulven Laurent décrit, enfin, l'originalité des conceptions du paléontologue Marcellin Boule (1861-1942) se démarquant de toute vision «évolutionniste » (Gabriel de Mortillet) et soutenant que la branche humaine, bien qu'indépendante depuis le début des autres branches de primates, $s$ 'est cependant développée parallèlement en demeurant morphologiquement et géographiquement " voisine ». 
Amor CheRn, Buffon. La nature et son histoire, Paris, Presses universitaires de France, 1998. 11,5 × 17,5, $136 \mathrm{p}$, index (Philosophie, 101).

Ce petit volume ne se propose bien évidemment pas d'exposer en détail les trente-six volumes de l'Histoire naturelle, mais de rendre compte du projet qui anime l'entreprise de Buffon, et plus précisément d'interoger le contenu de la notion d'histoire mobilisé dans cette ouvre. Une question centrale commande le développement de l'analyse, celle de l'origine du projet : à quoi tient l'émergence de l'histoire naturelle? Postulant dès les premières pages que l'histoire naturelle est science du devenir de la nature et naît avec l'ceuvre de Buffon, l'auteur installe une double ambiguité, que la suite de l'ouvrage vient heureusement dissiper. La thèse que Amor Cherni entreprend de soutenir est que « l'histoire naturelle n'est née que parce que la Nature est devenue moins visible aux yeux des hommes ", c'est-à-dire moins immédiatement proche de l'homme, en raison des nouveaux instruments, conceptuels et techniques, utilisés pour l'étudier. L'histoire naturelle se déployant pour Buffon à deux niveauxx, celui des choses inertes et celui des vivants, c'est la question de l'indépendance ou de la solidarité originaire de ces deux mondes qui se trouve posée. En d'autres termes, c'est la question de l'origine du vivant qu'il convient d'interroger, partant celle de la position de Buffon à l'égard des hypothèses concurrentes de la préformation et de l'épigenèse, donc aussi celle de la teneur de son matérialisme et de sa relation au mécanisme.

La brève introduction exposant la problématique d'ensemble de l'ouvrage est suivie de cinq chapitres, où l'auteur, loin de s'abandonner à une fastidieuse description ou de se perdre dans les détails de cette ceuvre monumentale, se propose de mettre à jour "ses modèles et ses fondements théoriques", et, pour cela, procède, en une progression fortement ordonnée, par interrogations successives guidant le lecteur et assurant une compréhension aisée par son style simple et concis.

Le premier chapitre, essentiellement centré sur le Premier discours, présente la réflexion méthodologique de Buffon; son exigence d'une méthode «naturelle ", apte à la fois à embrasser la multiplicité du vivant et à en saisir l'unité, son souci d'appliquer au «magasin de la nature », préalablement constitué, un regard où l'observation s'affranchit de l'arbitraire des systèmes. C'est en fait un double arbitraire que dénonce Buffon, arbitraire des systèmes de classification artificielle, conduisant à une "pléthore taxinomique ", arbitraire des mathématiques engendrant les limites du mécanisme. Contre le privilège reconnue à la méthode déductive et la prétention d'atteindre à une connaissance absolue de la nature, prétention illusoire puisqu'elle a consisté au contraire à penser la production naturelle à partir de nos propres productions, Buffon affirme la distance et l'extériorité qui sépare le sujet connaissant de l'objet qu'il étudie et les droits à valoir de l'observation se limitant à dégager les rapports entre les êtres et entre les choses. En conséquence, si Buffon se propose de mettre à jour la «méthode de bien conduire son esprit dans les sciences ", c'est moins pour suivre Descartes que pour dénoncer l'analyse comme voie royale et exclusive à l'intelligibilité du réel.

Le second chapitre est consacré à une présentation, claire et argumentée, des éléments de la théorie de la terre, insistant, à bon droit, sur le jeu dominant des « causes constantes " et sur l'hypothèse du choc par laquelle Buffon s'efforce de rendre compte du système solaire. L'étude du vivant, développée au chapitre suivant, 
accorde une attention particulière aux concepts centraux de moule intérieur, de forces pénétrantes et de molécules organiques, ce qui permet de situer la position de Buffon à l'égard de l'hypothèse préformationniste. Conjointement l'auteur montre pertinemment que la position de Buffon à l'égard du mécanisme ne se réduit pas à un simple rejet, mais constitue plutôt son élargissement, illustration de sa défiance à l'égard des généralisations simplificatrices et de «l'esprit de système ». Ces deux chapitres préparent en fait l'analyse de l'historicité de la nature inanimée et du vivant, du balancement entre génération et dégénération, qui met en lumière à la fois le rôle des considérations morales et théologiques dans la pensée de Buffon et l'évolution de sa représentation des rapports entre l'homme et la nature. L'auteur entend notamment montrer que «bien avant Charles Darwin, Buffon a vu non seulement l'importance de la domestication dans la variation des espèces, mais aussi le rapport d'analogie qu'il y a entre cette variation et celle qui se fait dans la Nature ». lls préparent, enfin, l'exposé de la réflexion de Buffon sur l'origine du monde, laquelle vient répondre aux questions liminaires pour montrer le poids du newtonianisme sur la pensée de Buffon et le rôle privilégié qu'il attribue à la lumière dans la naissance du monde, faisant apparaitte son histoire comme « le passage d'une Nature transparente à une Nature opaque ».

En définitive, Cherni s'efforce ici avec succès de mettre à jour les principes directeurs qui commandent le discours de Buffon. Sans doute, les difficultés que soulève l'analyse ne pouvaient, étant donné les exigences de la collection, recevoir les approfondissements nécessaires à leur complète intelligence. Mais, on aurait pu espérer que le lecteur novice auquel s'adresse l'ouvrage trouve quelques pistes bibliographiques afin de lui permettre de pousser plus loin son étude de la pensée de Buffon. L'absence de toute référence aux travaux de Jacques Roger est, à cet égard, étonnante.

Thierty MARTIN

Giuliana Gemelu, Le Élites della competenza. Scienziati sociali, istituzioni e cultura della democrazia industriale in Francia (1880-1945). Bologne, 11 Mulino, 1997. 13,5 × 21, 419 p., index (Ricerca).

Sauf à être dépositaire d'un capital social et symbolique particulièrement élevé, le lecteur du livre très fouillé de Giuliana Gemelli (qui n'a pas ménagé ses efforts côté sources tant en France qu'aux États-Unis) ne peut qu'être frappé d'un certain vertige tout au long de la rencontre que l'historienne transalpine nous propose avec cette page originale de l'histoire de France. $\grave{A}$ la fois analyse de certains des fondements du système des relations industrielles françaises et du développement de l'économie dirigée, examen des présupposés et des initiatives tant des téformistes sociaux que des premiers « technocrates $»$, la réflexion se donne pour toile de fond certaines des conceptions qui ont dominé l'histoire des rapports capital-travail au cours de la Troisième République (notamment sous l'influence américaine) et décline, d'une certaine manière, les rapports du savant, du politique et du capitaliste. L'ouvrage est 
de fait tout entier construit autour du rôle des sciences sociales dans l'édification et l'affirmation de la "démocratie industrielle * française. Quand on mesure aujourd'hui combien sont cruciales les questions tant theoriques que pratiques et politiques sur l'économique, la place de l'entreprise dans la société, le rôle du marché, les rapports État-marché, autrement dit les questions relatives à l'intervention de l'État, et quand bien même soufflent fort les vents du néolibéralisme, il importe que puissent être éclairés certains des éléments fondateurs d'un modèle de société qui a réussi à porter au pinacle de la démocratie les valeurs du marché et de l'entreprise. De ce point de vue, le rôle majeur rempli par les sciences sociales pour en rendre compte, l'éclairer et tantôt le justifier (ici principalement économie, statistique, sociologie, droit) doit pouvoir être étudié, et pour donner à comprendre les enjeux globaux de société et pour alimenter un retour réflexif et critique sur les sciences sociales elles-mêmes.

Si le lecteur est susceptible d'être frappé de vertige, cela tient non seulement à l'extraordinaire galerie de portraits de ces élites intellectuelles et stratégiques qui convolent de beaux mariages en hauts lieux (de l'administration d'État, des affaires et des univers académiques) et qui ont pour particularité d'être dépositaires (et souvent producteurs) des savoirs des sciences sociales. Mais aussi, ces élites se placent-elles au carrefour d'associations et d'organisations - institutions éducatives nouvelles, revues, structures hybrides de rencontre et d'action, mouvements de réflexion, etc. - qu'elles créent, impulsent et investissent. C'est dans ces franges du pouvoir d'État et des milieux patronaux, qu'ont été pensées les diverses doctrines sociales de gouvernement de la société française depuis le réformisme solidariste jusqu'aux fondements de la technocratie et de la planification soigneusement conçus par les polytechniciens des années trente en passant par la grande utopie rationalisatrice d'inspiration taylorienne. C'est là que l'historienne observe la singulière alchimie des élites intellectuelles administratives et économiques qui construisirent un langage tout entier arc-bouté sur le savoir des sciences sociales, le langage de la compétence et de l'expertise, fondement d'une véritable «syntaxe de l'action sociale » (et économique) ainsi que d'un authentique « territoire de l'autorité technique ", fruit de la rencontre de différentés cultures autour des problèmes de l'organisation, du monde de l'entreprise et des modes de prise en charge publique des relations industrielles. Ce faisant, ce sont l'organisation, les conditions de production et les objets de la recherche en sciences sociales qui évoluent et définissent peu à peu les contours d'un État industriel « moderne».

Dans la première partie de l'ouvrage, après avoir caractérisé la spécificité des rapports entre la science et l'État dans l'acception durkheimienne - une spécificité qui est très éloignée du modèle d'économie mixte de la recherche en Allemagne ou encore du modèle américain basé sur la prééminence de la grande entreprise et du corporate capitalism - Gemelli examine attentivement le rôle singulier des disciples et continuateuts de l'ceuvre du sociologue Frédéric Le Play. Plus précisément, c'est à une fraction modernisatrice des leplaysiens, catholiques sociaux, que l'auteur s'intéresse (Émile Cheysson, Paul de Rousiers, Max Leclerc ou Robert Pinot) et que l'on retrouvera surtout, en dehors des sphères académiques, dans des univers hybrides (relevant tantôt de l'État, tantôt du patronat) et en particulier au Musée social, créé en 1889 grâce au mécénat du comte de Chambrun. L'auteur avance par exemple que, durant le bref laps de temps où il dirigea le Musée social, Pinot 
impulsa « un modèle inédit de gestion d'une entreprise culturelle * (p. 81). Ni structure d'enseignement, ni cercle intellectuel mais plutôt "prototype des sociétés de conseil modernes », cette organisation est représentative d'un « modèle d'agrégation mixte, capable de réunir diverses élites dans l'objectif de diffuser une forme d'interventionnisme social scientifiquement fondé " ( $p .72$ ), en particulier par l'application rigoureuse des méthodes de recherche statistique. L'auteur examine ensuite les parcours et réalisations de deux hommes qui créeront, l'un - Victor Duruy - l'École pratique des hautes études en 1868, l'autre - Émile Boutmy - l'École libre des sciences politiques au lendemain de la défaite de Sedan. Sur cette demière initiative, soutenue par d'éminentes personnalités, Gemelli analyse comment, jusqu'à la création de l'École nationale d'administration en 1945, l'École libre exercera un rôle majeur dans l'organisation de la culture du monde des affaires, de la diplomatie et de la haute administration.

Dans la deuxième partie, l'auteur observe le noyau dur de ces groupes de réformateurs sociaux animé en particulier, dans le cadre des Entretiens de Pontigny, par Paul Desjardins, véritable «entrepreneur culturel » avant la lettre. $\grave{A}$ partir de $c e$ réseau, Gemelli examine le "caractère culturel pluraliste » d'autres associations créées à la même époque : l'Association internationale pour la protection légale des travailleurs (1906), l'Association pour la lutte contre le chômage créée à l'initiative de Max Lazard en 1910, etc. Avec la guerre, et en toile de fond le débat crucial sur l'organisation et la rationalisation - autrement dit entre la découverte enthousiaste des États-Unis et la valorisation concomitante de l'OST (organisation scientifique du travail) et l'obsession d'une opposition à l'Allemagne considérée comme détentrice du secret (et du savoir) de l'organisation - l'auteur entend montrer que le développement de l'expertise ne peut être lié exclusivement à l'expansion de l'interventionnisme étatique résultant de la guerre. C'est ainsi qu'elle s'intéresse de près aux initiatives organisationnelles impulsées depuis les comités consultatifs d'action économique (décret de 1915) ou au rôle important joué par Étienne Clémentel, ministre du Commerce et de l'Industrie, lequel s'appuya grandement sur le travail de conseil et d'expertise de l'historien économiste Henri Hauser qui élabora, en particulier, le concept de région économique. De même, Albert Thomas, ministre des Armements pendant la guerre, un normalien au parcours emblématique, s'entoura d'éminents scientifiques sociaux tels François Simiand, Maurice Halbwachs, Fernand Maurette, Paul Mantoux, etc. Pour mieux appréhender les logiques d'organisation des intétêts (tant professionnels que patronaux ou syndicaux), Gemelli analyse alors le contexte, les principaux points de discussion juridique ainsi que le rôle au demeurant ambivalent de la CGT réformiste de Léon Jouhaux dans le difficile avènement du Conseil national économique en 1925. Dans ce cadre, la dimension centrale de la compétence et de l'expertise - valorisée et théorisée par le juriste Joseph Barthélemy - est clairement mise en lumière.

Dans la dernière partie du livre, sont étudiếs tout une série de parcours représentatifs des normaliens et leurs rôles depuis les « laboratoires intellectuels de l'École normale supérieure " créés grâce à d'importants concours financiers (mécénat d'Albert Kahn, gros investissements de la fondation Rockefeller), et la véritable croissance des systèmes privés d'organisation de la recherche - expression de l'effervescence et de l'intérêt croissants pour les États-Unis dont témoigne la multiplication des voyages d'études des scientifiques sociaux outre-Atlantique. Mais, 
pour caractériser plus encore cette « autorité des experts », l'auteur étudie le rôle de l'Ecole polytechnique dans la formation de ce qu'elle appelle, faute de mieux, une véritable " socio-technocratie *. L'bistorienne s'intéresse ainsi à la redéfinition du rôle du polytechnicien en « ingénieur-économiste * (p. 309), et à d'importantes créations organisationnelles et associatives. Sont analysés - et pour cause - les effets et initiatives qui suivirent directement la tenue à Paris du premier congrès international de l'Organisation scientifique en 1925, à savoir, la création de l'Institut international du management qui diffusera largement les grands préceptes tayloriens, et le développement de la professionnalisation de l'expertise et des premiers cabinets-conseils (en organisation); c'est, enfin, une double création - du côté des milieux patronaux - animée par des polytechniciens: Henri Fayol au CNOF (Comité national de l'organisation française), Jean Coutrot et Jean Milhaud à la CEGOS (Commission générale d'organisation scientifique). Gemelli étudie également le rôle important de X-Crise, cette association loi 1901 (au depart Centre polytechnicien d'études économiques) qui favorisera le plus la réflexion sur le modèle de " l'Etat technique », à savoir \& un État qui valorise la compétence technique [...] présente dans toutes les institutions et dont le rôle primordial est précisément de les faire interagir, en ctéant un circuit d'informations permanent au service des institutions et de chaque citoyen $»$ (p. 339).

Avec la Seconde Guerre mondiale et la dissipation de l'intérêt des experts français pour le scientific management d'inspiration taylorienne liée aux « deux révolutions paradigmatiques » dans les modèles scientifiques américains (développement des behavior sciences et de l'operational research ou, plus généralement, le développement des modèles mathématiques appliqués à l'économie), l'auteur explore à la fois la transformation qui s'opère dans la nature des échanges franco-américains, les figures nouvelles, souvent inédites, de ces élites représentées exemplairement par des « administrateurs de la science » ou encore par des « éveilleurs " et des "colporteurs d'innovation » situés en des lieux stratégiques de part et d'autre de l'Atlantique, mais aussi les conséquences du développement de l'économétrie.

Incontestablement, on a affaire à un liwre dense et stimulant. Le regard extérieur, la perspective comparative France-États-Unis, cette singulière histoire des sciences sociales - dont on peut rappeler que leur autonomisation ne s'effectue que dans la seconde moitié du siècle - ne peuvent qu'intéresser bon nombre de chercheurs afín de rompre, comme l'écrit Pierre Bourdieu, avec "le rapport routinier à l'héritage " qui se nourrit de l'amnésie de la genèse. Mais, au-delà de la communauté des chercheurs et des difficiles débats qui les concernent aujourd'hui, ce livre intéressera un public plus large car, et l'enjeu est peut-être insuffisamment explicité, il contribue à alimenter l'analyse sociologique et historique de l'État. Même si l'on ne partage pas l'ensemble des thèses défendues, cet ouvrage mérite amplement d'être traduit.

Joseph Romano 
Artuto EscoBAr, Encountering development. The making and unmaking of the Third World. Princeton, NJ, Princeton University Press, 1995. 15,4 × 23,5, 291 p., notes, index (Princeton studies in culture/power/history).

Cet ouvrage d'un Colombien qui enseigne l'anthropologie à l'université du Massachusetts suscite une certaine curiosité. Il s'agit en effet, à partir d'une bibliographie étendue, d'une analyse du " discours du développement » inlassablement reproduit depuis un demi-siècle enjoignant aux pays d'Asie, d'Afrique et d'Amérique latine relevant du tiers-monde d'imiter l'exemple des pays industrialisés d'Europe et d'Amérique du Nord. L'inspiration en est expressément attribuée aux travaux de Michel Foucault sur la « dynamique du discours et du pouvoir dans la représentation de la réalité sociale $»$. Un certain nombre d'autres auteurs français sont également cités - Jean Baudrillard, Gilles Deleuze, René Girard, etc. (toujours en traduction).

Depuis la fin de la dernière guerre, le concept de développement économique et, le plus souvent, tout simplement de développement est effectivement d'une très grande généralité. An temps de la guerre froide, il êtait l'objectif visé par les deux côtés en présence, même si méthodes et résultats attendus différaient beaucoup. Peut-être jugera-t-on un jour qu'il fournit la meilleure illustration possible de l'idéologie de notre époque. D'où l'intérêt de l'examiner de près.

Développement et modernité (et l'effort de « modernisation $*$ ) sont tenus pour à peu près synonymes. À l'opposé, une ère « postmoderne " et de "postdéveloppement " est présentée à la fín de l'ouvrage comme restant aujourd'hui à imaginer. Dans le discours "postmoderne " (Jean-François Lyotard n'est pas cité), les êtres vivants ne sont plus conceptualisés en termes d'organismes organisés et localisés hiérarchiquement, mais en termes de systèmes codés de communication et de cybernétique, de réseaux de contrôle et de probabilité. Le postdéveloppement, défini par référence aux travaux d'un certain nombre d'analystes latino-américains, ne verra plus lui-même « des processus de modernisation opérant par la substitution de ce qui est modeme à ce qui est traditionnel, mais une modernité hybride caractérisée par des tentatives continuelles de rénovation, par une multiplicité de groupes assumant la charge d'une hétérogénéité multitemporelle propre à chaque secteur et pays » (p. 218). Il s'agit de s'arracher au processus par lequel la diffusion « de l'économie de marché a détruit les liens commuñautaires et privé des millions de personnes d'accès à la terre, à l'eau et à d'autres ressources " (p. 22), et de devenir, enfin, humain dans un paysage « posthumaniste et postmoderne ". \& L'anthropologie de la modemité en termes de cultures hybrides $*$ ne peut toutefois en aucun cas être tenue pour \& l'achèvement du projet de la modernité » du temps des Lumières auquel invite Jürgen Habermas dans Le Discours philosophique de la modernité (trad. franç., Paris, Gallimard, 1988) : l'application des efforts de développement aux pays du tiers-monde, qui en est la demière tentative, en montre précisément l'échec (p. 221).

Le discours du développement en question est le message qui accompagne l'expansion du capitalisme contemporain. Sa « déconstruction " est recherchée. Cependant, Jacques Derrida, généralement tenu pour l'auteur de ce concept, n'est pas cité (sauf à travers une référence critique de Habermas), et le terme lui-même 
n'est pas explicité. Ou du moins son sens de dévoilement d'une hiérarchisation cachée ne ressort qu'indirectement, à travers, par exemple, la dénonciation rituelle que font d'innombrables travaux de la « pauvreté » des pays « les moins développés ". Une autre utilisation du concept peut être vue dans la référence aux économistes réformistes des années trente et quarante qui furent les pionniers de l'application de la notion de developpement au domaine nouvellement constitué du tiers-monde : ceux-ci sont présentés avec plus de sympathie que les économistes néolibéraux des années quatre-vingt mais leur vision de la "planification du développement , décrite comme très théorique et utopique, est traitée de la même façon (chap. m).

Compte tenu de l'optique adoptée, on aurait pu s'attendre à un examen attentif de l'origine et du contenu de la notion de développement. Il ne sera pas reproché à l'auteur de ne pas l'avoir tenté, puisque son propos n'était pas de plonger dans l'imaginaire des pays « développés ». Mais il s'est privé ainsi d'une orientation qui l'aurait aidé à préciser sa critique. Le mot de développement, en effet, a commencé

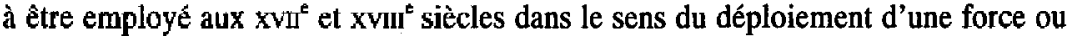
valeur. Il a été défini de façon savante comme « la mise au jour, par croissance graduelle dans l'espace et le temps, d'une structure préétablie présente dès l'origine d'un être ou d'un processus en un germe invisible qui en contient le programme » (voir Entre forme et histoire. La formation de la notion de développement à l'âge classique, Paris, Méridiens Klincksieck, 1990). S'il n'est appliqué qu'au xx siècle au développement physiologique et psychologique d'un être et au développement économique, il l'est dès le temps des Lumières, en tant que déploiement de virtualités, à l'histoire et à la vie de l'homme et des sociétés. Ainsi vont naître l'idée du développement de chaque individu, du " développement de ses facultés morales et physiques » (travaux préliminaires de la Déclaration de 1789), du « développement des dispositions naturelles des hommes " (Kant, L'Idée d'une histoire universelle au point de vue cosmogonique, 1784), celle de « développer toutes leurs facultés naturelles" (Condorcet, Esquisse d'un tableau historique des progrès de l'esprit humain, 1794), ou le principe directeur reconnaissant «l'importance absolue et essentielle du développement humain dans sa plus riche diversité " (Wilhelm von Humboldt, 1792, cité par John S. Mill en exergue à son livre On liberty, 1859).

L'auteur sans doute n'est pas le seul chez qui on peut relever cette omission. Mais cette référence aurait permis de préciser que si, dans le domaine des sciences humaines, l'idée de développement s'applique avant tout au déploiement des virtualités qui sont celles de chaque individu, l'application au đéveloppement économique ou politique d'un ensemble social ne représente, par analogie, que le moyen devant permettre la réalisation de ce développement humain. Si donc les transformations économico-sociales auxquelles on s'attache depuis les années quarante n'y conduisent pas, il y raison de les critiquer. Mais peut-être dans ce cas devrait-on surtout les désigner par un autre terme que celui de " développement".

L'intérêt de l'ouvrage tient en fait avant tout aux analyses concrètes des politiques dites de développement qu'il contient. Ainsi sont longuement présentés les programmes de développement rural préparés ou soutenus, à l'usage des pays du tiers-monde en particulier d'Amérique latine, par les organisations internationales et notamment la Banque mondiale. Leurs effets sont décrits comme plus que douteux. Les paysans se sont vus offrir des " paquets technologiques ", semences améliorées, 
pesticides, concentration sur certaines productions, préparation de plans de production détaillés en vue de mises sur le marché, etc. Cependant, si le nombre de ces paysans a diminué pendant que se produisait un exode massif vers les villes, leur très grande majorité est restée pauvre et soumise à des relations archaïques de domination. Les efforts gouvemementaux ne changent pas grand-chose, et la masse des ressources financières, technologiques et intellectuelles consacrées à l'agriculture continuent à se diriger vers le secteur capitaliste (p. 150) - ce qui n'a pas empêché une forte augmentation de $l^{\prime}$ importation de produits agricoles et alimentaires.

La réalité des échecs rencontrés est indéniable. Le nombre des études qui ont contribué à la faire apparaître est impressionant. Sans doute est-il difficile de bien établir une relation de cause à effet entre les politiques suivies et ces résultats négatifs. Arturo Escobar s'attache surtout à montrer le non-dit qui se laisse deviner derrière tous les documents qu'il étudie, et là il marque des points. Il en marque d'autres lorsqu'il fait apercevoir l'étroitesse intellectuelle de beaucoup des analyses aujourd'hui conduites au service $d u$ « développement $*$ On demandera néanmoins en terminant s'il ne s'agit pas d'une autre forme de discours lorsqu'il est avancé que la * production de discours dans des conditions de pouvoir inégal * - schéma effectivement courant - est elle-même une condition suffisante de sa propre pérennité.

Philippe J. BERnarD

Francis Affergan, La Pluralité des mondes. Vers une autre anthropologie. Paris, Albin Michel, 1997. 14,5 × 22,5, 295 p., bibliogr. (Bibliothèque Albin Michel Idées).

Selon Francis Affergan, professeur d'ethnologie à l'université de Nice et spécialiste des Antilles, l'anthropologie française est en crise : du côté du terrain, les modes de vie occidentaux s'étant diffusés partout, l'anthropologue n'a plus pour objet d'étude des peuples biens délimités qui seraient à l'abri des métissages; du côté de la théorie, la tentation de formaliser les soçiétés à l'aide de modèles nomologiques, holistes, et de lois extensionnelles, pour conférer à l'anthropologie la même rigueur que les sciences de la nature, obéit à un leurre dangereux ou stérile.

L'objet du livre est de réarticuler le terrain et la théorie. Le terrain ne relève pas d'une observation neutre, mais déjà de la construction d'un « monde-entre ", d'un chiasme entre le monde de l'observateur et celui de l'observé. Quant à la théorie, pour la sortir des illusions scientistes et de la logique classificatoire que promeut la matricielle ethnologique de la parenté, Affergan s'inspire à la fois du schème kantien, de l'esquisse husserlienne, et de l'exemple type wittgensteinien, pour proposer l'idée d'une construction schématisante et simultanée de l'objet et de sa connaissance, « dans la mesure où les distinguer conduisait immanquablement à oblitérer soit le travail d'investigation empirique, soit sa recomposition interprétative ».

Il faut pour cela sortir du comparatisme - « lorsqu'on ne sait pas interpréter, on compare ». Montrer les présupposés du modèle de la parenté qui fonctionne comme 
une machine décrivant la transmission de constantes, spatialise le phénomène et masque son événementialité. Déconstruire les universaux ethnologiques qui prédécoupent le réel (le clan, le lignage, la tribu, etc.), et les doublets à la fois géographiques et épistémologiques produits par la théorie ethnologique (la hiérarchisation en Inde, l'honneur et la honte en Méditerranée, la piété filiale en Chine, le lignage et le segment en Afrique, etc.). Concevoir des modèles qui ne copient plus le réel mais créent des mondes possibles à la manière de l'cuvre d'art, qui s'en éloigne pour mieux le connaître. Voilà ce à quoi s'attache Affergan dans un premier temps, contre les traditions néodurkheimienne et structuraliste qui passaient sous silence les aspects «constructifs, problématiques, imaginaires et temporalisant $»$ de la pratique anthropologique. C'est en introduisant les notions de personne et de découverte, qu'Affergan fait une place, au sein de l'anthropologie, à l'étude des valeurs et de la temporalité. La personne remplace l'individu : au lieu d'être un quoi interchangeable dans une logique des places et des commutations, elle est un qui répondant de ce qu'il fait dans un dialogue infini. D'autre part, l'ethnologue n'observe pas, mais « découvre au sens où il invente ": il découvre ce dont il a besoin pour fonder la pertinence de son monde, il n'utilise pas des preuves mais des arguments, son objet relève de la forme, au sens du schématisme kantien, et non de la chose. Cela dessine une épistémologie «à la fois génétique et évolutive » dans laquelle le contexte de découverte ne nuit en rien au contexte de justification.

Affergan dresse ensuite des typologies de modèles possibles, qui doivent leur fécondité à leur capacité de montrer des mondes, et non pas de satisfaire l'expression algorithmique d'une loi extensionnelle. Il montre que la traductibilité des cultures se fonde sur une intraductibilité fondamentale : les traductions sont toujours des tentatives de traductions. Puisqu'il faut rompre avec l'idée mécaniste qui fait se correspondre terme à terme les mots de deux langues et qui identifie le mot, l'idée et le segment de réalité, traduire s'assimile à l'acte de rendre compte des échos que portent les mots, ce qui revient à produire un nouveau monde, et l'on s'aperçoit que le travail de l'anthropologue est bien celui d'un traducteur de cultures.

Enfin, prenant appui sur son propre terrain antillais, Affergan montre la fécondité des notions de fiction et d'événement en anthropologie. Une ethnohistoire a beaucoup de mal à se constituer aux Antilles pour plusieurs raisons : absence d'histoire de longue durée, de profondeur spatiale et d'arrière-pays, vitesse de la colonisation, oubli des racines, et réussite du modèle assimilationniste. Manque d'histoire qui se traduit par le sentiment d'être face à une société artificielle (un « laboratoire $»$ ), une société qui n'a pour tradition que les fictions qu'elle se raconte à elle-même, société carnavalesque (au sens de Bakhtine), dont l'identité est déguisée, ou mieux, dont l'identité est le déguisement-même. N'est-ce pas ce qui caractérise nos mondes de plus en plus métissés, marqués à la fois par des événements castrateurs de mémoire (déportation, colonisation) et des « transplantations historiques »? L'anthropologie de la modernité n'est-elle pas celle des collages surréalistes, de l'incongruité, du bricolage et du fragment?

Dans la construction d'un monde et d'une mémoire, l'événement n'est pas un fait qui se produit, mais ce qui est repris dans une narration. L'événement n'est pas ce qui arrive, ce qui va de soi, mais ce qu'on fait arriver et qui se pense comme un obstacle à construire; Affergan s'inspire en cela d'Aristote, de Paul Ricour et de Reinhart Koselleck, pour bâtir une anthropologie qui serait « sous la double égide de la 
fiction, comme acceptation de mondes en constructions, et de l'événement, comme indice d'une temporalité sémantique $»$.

Même si ce livre a parfois l'aspect d'un catalogue de références (pas toujours très approfondies) et si l'exemple antillais semble trop typé ou trop effleuré pour pouvoir refonder d'un coup l'épistémologie de l'anthropologie, des pistes essentielles sont néanmoins tracées qui donnent à penser les linéaments d'une * autre anthropologie $\$$.

Céline Jouın

Bert Hölunobler et Edward O. WILson, Voyage chez les fourmis. Une exploration scientifique. Trad. de l'américain par David Ouvier. Paris, Seuil, 1996. $19,5 \times 24,250 \mathrm{p}$., index, ill. (Science ouverte).

Les myrmécologues apprécieront le contenu scientifique de ce livre. La renommée des auteurs dans ce domaine, la collaboration d'un spécialiste français à la traduction, la brève mais élogieuse recension de l'ouvrage dans les colonnes de $L a$ Recherche (294, janv. 1997, p. 104), laissent penser qu'ils le jugeront favorablement.

Cela étant, ce n'est pas à eux que les auteurs destinent en priorité leur livte. Ils s'en expliquent dès les premières lignes : surpris eux-mêmes par le retentissement auprès du public de The Ants, volumineuse et austère monographie parue en 1990, ils ont décidé d'offrir au lecteur un condensé du ameilteur de la myrmécologie en un volume plus raisonnable, avec moins de jargon technique $m$. Tout se passe donc comme si les auteurs, après avoir écrit un ouvrage pour les chercheurs, en avaient donné une version plus brève et plus attractive à l'usage du public. $\hat{A}$ ce titre, il mérite de retenir l'attention de tous ceux qui s'intéressent à la vulgarisation scientifique. Qu'on se reporte, en particulier, à l'étude magistrale d'Yves Jeanneret, Écrire la science (Paris, Presses universitaires de France, 1994), ou à la présentation du numéro spécial de la Revue de synthèse intitulé «Animalité et anthropomorphisme dans la diffusion et la vulgarisation scientifiques * (t. CXIII, 3-4, 1992), et l'on verra combien plusieurs des traits caractéristiques de la vulgarisation se retrouvent ici.

Plus nettement encote que le titre français, le titre original Journey to the ants. $A$ story of scientific exploration révèle le désir de séduire le lecteur par l'attrait de la narration. Effectivement, de multiples anecdotes - généralement autobiographiques et toujours en rapport avec des travaux de recherche sur les fourmis -

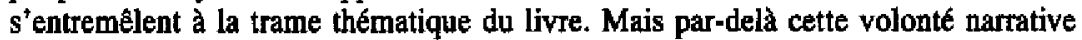
inhérente à toute cuvre de vulgarisation, le titre, par l'évocation d'un \& voyage *, nous place d'emblée dans l'univers de la fiction thétorique. Car, s'il est question de déplacements dans ce livre, ce sont ceux qui ont permis aux deux auteurs d'aller dans diverses régions du globe observer différentes populations de fourmis. Le périple ici ne peut être singulier que par une métaphore qui suggère que les fourmis vivent dans un monde à la fois proche et éloigne du nôtre et dont l'exploration - 
patiente et difficile - peut être source d'excitation intellectuelle. C'est là un thème qu'on trouve déjà en 1798 sous la plume de Pierre-André Latreille dans l'Essai sur l'histoire des fourmis de la France (voir Revue de synthèse, t. CXIII, 3-4, 1992, p. 333-345). Cet artifice rhétorique peut sembler heureux ou convenu mais il est sans conséquence quant à l'efficacité didactique. Il n'en est pas de même du thème qui lui est souvent associé : celui de la taille. Quel meilleur moyen en apparence de faire comprendre au lecteur l'importance des fourmilières que de les rapporter à son échelle. Ainsi (p. 132-133), à propos d'un nid d'une espèce de fourmi défoliatrice du Brésil (Alta sexdens), les auteurs indiquent que sa construction « équivaut bien à l'écheile humaine à la construction de la Grande Muraille de Chine \%. Or, on sait depuis longtemps que ce genre d'analogie est critiquable puisque la force d'un animal est proportionnelle au carré de sa dimension tandis que les masses varient comme le cube des dimensions. Bert Hölldobler et Edward Wilson qui, quatre pages auparavant, ont égratigné Maeterlinck pour sa conception mystique de « l'esprit de la ruche ", auraient été inspirés de suivre la prudence dont il fait preuve, en 1930, dans La Vie des fourmis sur le thème des dimensions.

Plus difficile à éviter et de tout autre conséquence, une autre source de confusion dans la littérature de vulgarisation sur les fourmis tient à leur caractère social. En l'occurence, la question est d'autant plus sensible que Wilson est, comme le rappelle la quatrième de couverture « connu pour ses théories sociobiologiques fort discutées $\%$. Et il est vrai que le texte est émaillé de quelques perles. Ainsi le $\alpha$ mode de vie colonial hautement développé $*$ des fourmis inspire aux auteurs cette audacieuse conclusion : " Le socialisme, semble-t-il, fonctionne effectivement dans certaines conditions. Karl Marx s'est simplement trompề d'espèce » (p. 19). Ailleurs, il est suggéré que les fourmis qui abandonnent leur mère pour se rallier aux vainqueurs dans un combat pratiquent * l'équivalent fourmilier $*$ de la devise * mieux vaut rouge que mott (p. 105), allusion probable aux mouvements pacifistes allemands des années 1980. Au total, ces quelques remarques éparses passeraient sous la plume d'un autre auteur pour une tentative, raté, de faire de l'humour politique. Il serait imprudent de leur donner trop de portée. D'autant qu'en d'autres passages, Hölldobler et Wilson manifestent une indéniable volonté de rigueur terminologique, soulignant par exemple le caractère impropre du terme " esclavage " pour désigner une relation qui lie des fourmis d'espèces différentes (p. 144).

Il reste que les auteurs accordent une grande valeur à l'analogie entre des réalités biologiques distinctes: reprenant l'idé déjà ancienne de la colonie comme un superorganisme, ils ambitionnent de trouver dans la sociogenèse un éclairage sur la morphogenèse, autrement dit d'articuler l'étude des sociétés d'insectes avec la biologie du développement pour mieux comprendre les « principes généraux de l'organisation biologique (p. 129). Or, comme l'a montré Michel Veuille, dans son livre La Sociobiologie (Paris, Presses universitaires de France, 1986, p. 21-22), la limite de la sociobiologie réside dans son insistance sur " les mécanismes de l'évolution, plutôt que sur la phylogénèse, ce qui lui permet de mettre en valeur, en matière de comportement, les analogies entre espèces plutôt que les homologies $\%$. En d'autres termes, la société des fourmis peut être tapportée à celle des hormmes comme l'aile de l'insecte à celle de l'oiseau et non pas comme cette dernière à la jambe du cheval. En utilisant une autre métaphore, on pourrait dire que la nature a inventé plusieurs fois le fait social, mais de manière indépendante et avec des modalités bien 
différentes. À cet égard, on se reportera avec profit à l'analyse de Charles Lenay dans son compte rendu du livre de Pierre Jaisson, La Fourmi et le sociobiologiste (Paris, Odile Jacob, 1993) paru dans la Revue de synthèse (t. CXV, 1-2, 1994, p. 258-260).

Mais le souci principal des auteurs n'était visiblement pas là. Ce qui se dégage avant tout de la lecture de ce livre, c'est le désir de défendre et d'illustrer la myrmécologie, de montrer la place qu'occupe cette discipline au sein des sciences biologiques, la manière dont elle combine la systématique et l'éthologie, la biochimie et l'écologie. En témoigne, en particulier, l'insertion d'un chapitre entier tiré de The Ants et consacré aux techniques de capture, de mise en collection et d'élevage des fourmis (p. 227-242). Enfin, l'illustration, d'un réalisme didactique avec une certaine recherche esthetisante est également empruntée à The Ants. Ces deux emprunts marquent la volonté de réduire les distances entre le simple curieux, l'entomologiste amateur et le chercheur professionnel. Par là, ce livre atteste de la capacité des savoirs naturalistes à toucher un large public sans renoncer pour autant à une certaine technicité méthodologique.

Jean-Marc Drouin

«Observer l'animal », dossier établi et présenté par Frédéric Jouluan, Gradhiva. Revue d'histoire et d'archives de l'anthropologie, 25, 1999.

Le premier article de ce dossier est celui de l'éthologue Jacques Gervet. Il tente de déterminer quelles sont les diverses formes de causalité que les éthologues ont utilisées dans leur pratiques scientifiques, et d'en esquisser une histoire. Gervet avance une idée intéressante. Il estime que ce qui rend une discipline vraiment scientifique est l'introduction d'une forme spécifique de causalité. Il considère ainsi que George Bohn est le véritable père de l'éthologie scientifique avec la parution de son livre La Nouvelle Psychologie animale en 1911. Bohn y énonce deux propositions essentielles. Il réclame, tout d'abord, l'autonomie de la psychologie animale vis-à-vis de toute causalité strictement biologique. Il exclut, ensuite, la pertinence des psychologies du sens commun pour se substituer à une biologie peu satisfaisante. Mais comment faire cette histoire des causalités en éthologie? Gervet en esquisse les étapes qu'il juge importantes. Après la réponse behaviouriste, basée sur l'arc réflexe stimulus/réponse, il situe la synthèse objectiviste, celle de Konrad Lorenz et Nikolaas Tinbergen, qui introduit la « force motivationnelle w au creur du dispositif explicatif des comportements de l'animal. C'est à la suite de l'abandon de ce modèle physiologique de type énergétique, que Gervet place l'émergence d'une approche fonctionnelle, d'une part, et celle d'une approche mentaliste, d'autre part. L'approche fonctionnelle attribue un rôle central à la fonction adaptative du comportement. L'éthologie devient alors une écologie comportementale. Les modèles mentalistes, eux, sont doubles: on y trouve une approche subjectiviste d'une part, et une approche cognitiviste d'autre part. La première est récurrente, mais Gervet évoque surtout Jacob von Uexküll et Frederick Buytendijk, même s'il 
la retrouve chez un neurobiologiste contemporain comme Francesco Varela. L'approche cognitiviste est liée aux progrès nouveaux de l'informatique. Pour celle-ci, l'animal est confronté à des problèmes qu'il doit résoudre par l'intermédiaire de ses représentations de l'environnement. S'attaquer à un tel projet en l'espace de quelques pages est assurément un tour de force. Gervet l'assume très bien, même si on pourrait lui reprocher de trop rester au niveau de l'histoire des idées. On peut, en effet, discuter son analyse, en trouvant qu'il sous-estime trop la matérialité des pratiques de la recherche (l'ordinateur a considérablement transformé l'éthologie, mais sans doute plus comme outil que comme modèle), ou la révolution qu'a constituée la volonté d'aller observer des animaux sauvages dans leur milieu naturel pendant de longues périodes de temps (ce que ne faisaient pas les éthologues avant les années soixante). Enfin, de nombreux chercheurs ne sont jamais entrés dans ces schémas, comme Gregory Bateson, sans doute l'un des éthologues les plus intéressants du siècle.

Florence Burgat discute, quant à elle, la notion d'affordance, élaborée par le psychologue James J. Gibson en 1979, pour rendre compte de la perception du sujet, en insistant sur l'idée que l'environnement dans lequel agit celui-ci est déterminé par son usage de certains des éléments qui le composent. " Ce concept fait explicitement référence à la valeur utile des aspects de l'environnement vers lesquels la perception se dirige, et vaut pour l'homme comme pour l'animal. Il permet de décrire l'environnement comme une réalité agie » (p. 72). Burgat montre les parentés entre ce concept et les approches développées par les psychologues de la forme (Kurt Koffka et Kurt Lewin), par le biologiste von Uexküll avec ses concepts d'Umwelt, de Merkwelt et de Wirckwelt, et par le psychologue néerlandais Buytendijk. Elle essaie de montrer que, même s'il n'existe aucune filiation entre ces courants et Gibson, tous ont un ennemi commun, la psychologie objective. Pour Burgat, celle-ci équivaut à une psychologie behaviouriste qui s'appuie sur le dualisme cartésien du corps et de l'esprit, et qui refuse toute référence à la moindre notion d'intentionnalite. Burgat montre avec finesse que l'un des points clef du behaviourisme est la place privilégiée qu'y occupe le laboratoire, ainsi que le refus de toute approche environnementale de l'étude des comportements. Elle sous-estime cependant beaucoup notre propension à percevoir le behaviourisme à travers les lunettes d'un cognitivisme tout aussi agressif, qui a repris en douce quelques-unes de ses principales notions en les appelant autrement, et qui a artificiellement exagéré les différences entre les deux approches pour mieux faire ressortir une originalité sans doute moins grande qu'on ne veut nous le faire croire. N'oublions pas l'hostilité franche que reçoit encore toute approche écologique de la cognition au sein des sciences cognitives actuelles, et le succès remporté périodiquement par des approches néobehaviouristes comme le néoconnexionnisme ou les théories de la complexité au sein de ces disciplines.

Frédéric Joulian aborde le thème fascinant de l'habituation. « Les observations éthologiques modernes reposent en fait sur une relation nouvelle que les observateurs ont instaurée avec les animaux et qui leur permet de se faire accepter a leur côté : "l'habituation" " (p. 79). L'article souligne justement l'importance non seulement méthodologique, mais également conceptuelle de cette relation étrange qui 
s'instaure en éthologie entre un animal et un humain « pour la plus grande gloire de l'esprit humain " - tout au moins aimerait-on le croire, et l'expression prend toute son ambiguilté dans ce contexte où il s'agit justement de mieux comprendre l'esprit de l'animal! Même si on peut les trouver sommaires, on peut suivre Joulian quand il distingue trois situations spatiales et relationnelles entre l'homme et l'animal : celle de la séparation nette, celle de l'intersection et du recoupement des espaces, et celle de l'inclusion dans laquelle l'animal est sous la dépendance de l'homme. On peut également apprécier les nombreux exemples auxquels il se réfère. Inversement, que d'approximations, de contradictions, de bizarreries, d'occasions ratées et de non sens. Cet article pourrait presque devenir un morceau d'anthologie de ce point de vue. Qu'est-ce que "le vouloir du groupe animal »? Peut-on écrire à deux pages d'intervalle que l'étude des animaux captifs tient surtout de la paresse, et citer des résultats expérimentaux comme apportant des informations essentielles? On pourrait continuer longtemps ce petit jeu un peu futile. Joulian aborde pourtant quelques points intéressants. Dans le cadre de ce compte rendu, un seul sera examiné : Joulian s'engage, en effet, dans un débat essentiel, celui de la caractérisation du type de relation qui s'instaure entre l'homme et l'animal dans une situation d'éthologie de terrain. Mais son essai n'est pas satisfaisant, parce qu'il néglige de distinguer deux situations pourtant très différentes : celle de l'habituation de l'animal vis-à-vis de l'homme qui l'observe, et celle de la familiarisation de l'éthologue vis-à-vis de son sujet d'étude. S'il est important d'habituer l'animal à sa présence, non seulement de l'empêcher de fuir, mais aussi et surtout de se rendre transparent à ses sens, il l'est tout autant d'apprendre à le voir. Le phénomène a deux faces, complémentaires et symétriques. L'éthologue doit se faire de plus en plus transparent vis-à-vis de l'animal pour permettre à l'animal de se faire au contraire de plus en plus présent, èt d'acquérir une concrétude qui permette justement de comprendre non seulement ce qu'est l'animal, mais qui il est. La longue fréquentation des singes sauvages les plus complexes les personnalise. La transparence recherchée par l'éthologue de l'homme à l'animal s'effectue elle-même en deux temps au moins. Dans un premier temps, celui auquel Joulian fait le plus souvent référence, l'éthologue doit se faire le moins hostile possible pour l'animal. Mais dans un deuxième temps, il doit également se faire le moins amical! La tendance de l'animal sera d'introduire l'observateur humain dans son jeu, et d'en faire un allié, après s'être rendu compte qu'il n'était pas un ennemi. Le texte du primatologue suisse Hans Kummer, auquel se réfère pourtant Joulian sans citer ce passage, le dit presque explicitement. Kummer raconte, en effet, sa stupéfaction quand il se rend compte que les babouins qu'il étudie se servent de lui dans des stratégies complexes pour se protéger dés bergers éthiopiens qui les chassent! Enfin, il y a toujours un absent majeur dans ces rapports de terrain : l'assistant local. Ce n'est d'ailleurs pas tout à fait vrai. L'une des photos de Joulian montre, au milieu de babouins, ce qui doit être un assistant avec les singes. De dos. Une photo qui est presque plus parlante que le texte.

Le contraste est d'autant plus net avec l'article de Véronique Servais, qui aborde dans toute sa complexité, cette familiarisation de l'homme avec l'animal. Elle discute quelques-unes des difficultés qu'elle a rencontrées au cours d'une étude des relations thérapeutiques d'enfants autistes avec des dauphins. La question qu'elle pose est simple : les dauphins avec lesquels interagissent ces enfants améliorent-ils 
la condition de ces derniers? La démonstration de Servais est lumineuse et subtile : il ne peut y avoir de réponse non ambiguë à cette question, et ce d'autant moins que la réponse donnée aũa une influence sur les résultats escomptés. Les chercheurs rationnels n'ont pas nécessairement raison contre les thérapeutes New Age. L'implication émotionnelle de l'humain dans ses relations avec l'animal peut en être une dimension essentielle et en modifier les résultats obtenus. Le processus d'objectivation peut recéler des effets pervers contre-productifs. "Ainsi, s'il est vrai que les exigences de neutralité, de contrôle strict des variables et de reproduction conduisent à empêcher les changements apportés par la présence d'un animal de se produire c'est que la sensibilité à l'émotion et, probablement, au plaisir et à la beauté, c'est-à-dire tout l'imaginaire attaché à l'animal sont, non pas des illusions dont il faudrait se prémunir absolument, mais des ingrédients essentiels de la zoothérapie $»($ p. 105).

Il faudrait revenir plus longuement sur l'une des questions implicites les plus intéressantes soulevée dans ce dossier : qu'est-ce qui est différent dans l'observation de l'humain et dans celle de l'animal? Il serait sans doute audacieux de vouloir répondre d'emblée à une telle question. Quelques éléments de réponses pourraient cependant être discutés. D'abord, en évoquant ce truisme, sans doute tellement évident qu'il n'est jamais vraiment abordé dans le dossier, que l'homme parle et non l'animal. Ensuite, en rappelant cette vérité, pourtant contestée par certains, que la relation entre l'homme et l'animal est toujours asymétrique. En attirant l'attention, de surcroit, sur le fait que ce n'est pas l'« homme * qui observe l'animal, mais que ce sont des collectivités humaines qui s'intéressent à lui, en particulier par l'intermédiaire de techniques d'études et d'outils. En insistant, enfin, sur la situation très particulière de l'animal. Celui-ci n'est justement pas une machine, même si l'humain peut être leurré par certains artefacts ou bien s'en persuader (et chaque mot compte ici). Il recèle, au contraire, une insondable profondeur que l'humain a le sentiment de seulement effleurer. L'enjeu de ces interactions est finalement celui de l'identité spécifique de l'homme plus qu'autre chose.

Dominique LESTRL 